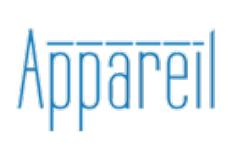

Appareil

$20 \mid 2018$

Arts contemporains et patrimoine

\title{
Situation : les paradoxes de la commande artistique - Jochen Gerz
}

Jean-Louis Déotte

\section{(2) OpenEdition}

1 Journals

\section{Édition électronique}

URL : http://journals.openedition.org/appareil/2713

DOI : 10.4000/appareil.2713

ISSN : 2101-0714

Éditeur

MSH Paris Nord

Référence électronique

Jean-Louis Déotte, «Situation : les paradoxes de la commande artistique - Jochen Gerz », Appareil [En ligne], 20 | 2018, mis en ligne le 23 octobre 2018, consulté le 30 juillet 2020. URL : http://

journals.openedition.org/appareil/2713 ; DOI : https://doi.org/10.4000/appareil.2713

Ce document a été généré automatiquement le 30 juillet 2020.

\section{(c) $($ ) $(9)$}

Appareil est mis à disposition selon les termes de la Licence Creative Commons Attribution - Pas d'Utilisation Commerciale - Pas de Modification 4.0 International. 


\title{
Situation : les paradoxes de la commande artistique - Jochen Gerz
}

\author{
Jean-Louis Déotte
}

1 Chez les Romains de l'Antiquité, lors de la naissance d'un enfant, celui-ci était exposé aux pieds du père. Dès lors, ce dernier, qui pouvait avoir des doutes sur sa paternité, considérait l'enfant encore tout ensanglanté gisant à ses pieds, et s'il pensait que sa légitimité n'était pas en jeu, ou si le nouveau-né n'était pas stigmatisé, il s'abaissait vers lui, le prenait dans ses mains et l'élevait au-dessus de lui, en signe de reconnaissance. Dans le cas contraire, l'enfant restait exposé et placé sur les marches d'un temple où il pouvait être adopté par d'autres parents ou récupéré par un marchand d'esclaves.

Le geste du pater familias, consistant à élever au-dessus de sa tête un enfant, aux yeux de tous, est à l'origine du fait d'élever un enfant, et le modèle de la reconnaissance d'une œuvre d'art.

On a donc là l'exemple classique d'une instance légitimante, car l'artiste et l'écrivain exposent aux pieds du public une œuvre qui, si elle est véritablement nouvelle, est par définition non reconnue, voire invisible, illisible. Ce qui change avec la «modernité ", c'est qu'il n'y a plus d'instance légitimante déjà constituée : il n'y a pas de public constitué, hormis les académies qui ne prennent en compte qu'un art préprogrammé. Ce qui est d'autant plus vrai s'il n'y a pas eu de commande : l'artiste expose à ses risques et périls. L'émergence d'une œuvre inconnue devient possible si celle-ci réussit à sensibiliser un public qui deviendra son public : elle va faire surgir peu à peu un public là où il n'y avait qu'une masse d'individus indifférents. Ce public va en retour la légitimer. Ce public va se constituer du fait qu'il élève l'œuvre. En l'absence de commande, il y a donc un nœud liant sensibilisation et élévation. C'est la situation ouverte par la "modernité ${ }^{1}$, au seuil de laquelle se tiennent Manet et Cézanne. Considérons les années nécessaires pour que La Recherche de Proust sensibilise son public et qu'en retour ce dernier lui donne une portée universelle. J'insiste sur ce nœud qui fait qu'il y a une œuvre pour un public, parce que l'œuvre et le public se constituent en face-à-face. Il faudra bien du temps pour que ce qui n'avait jamais été vu ou lu trouve une surface d'accueil. Il faudra sensibiliser cette surface pour que l'événement 
soit reconnu comme tel. Face à l'événement, la surface d'accueil qu'on appelle culture est intrinsèquement démunie, voire heurtée, elle réagira négativement plutôt qu'affirmativement. Dès lors le «Non!» du refus est bien davantage le signe de l'événementialité de l'œuvre que ne l'est le «Oui!». Tout simplement parce que la sensibilité commune est appareillée, et qu'un appareil sélectionne a priori ce qu'il peut accueillir et enregistrer. Et il rejette le reste. Ce reste, qu'une époque ne peut voir, penser et concevoir, appelons-le sublime. Peut-être qu'une autre époque, autrement appareillée, pourra l'accepter. Le sublime est donc relatif, historiquement parlant ${ }^{2}$ : autour de l'époque, il ouvre un halo qui est la ressource de l'avenir.

3 C'est la raison pour laquelle il faut affirmer qu'il y a toujours nécessité de la commande pour la production littéraire, philosophique et artistique. La commande suppose toujours l'intervention d'un autre ou d'un grand Autre, comme aurait dit Lyotard : celui qui exige de vous que vous sortiez de vous. Celui grâce à qui on va sortir de ses petites obsessions, de la répétition des choses bien connues et bien maîtrisées, bien faites, où l'on est bien chez soi. La commande, c'est ce qui perce le désir trop contrôlé, celui qui amènerait à une production qui se connaît bien, sans surprises. La commande percerait les couches de ce désir, de cette volonté d'auto-reproduction dans le cadre d'un narcissisme bien accepté, qui ne pose pas de problèmes. La commande briserait ces couches superficielles pour atteindre des couches " inférieures ", disons " archaïques ». Elle serait alors l'occasion de l'apparition d'une œuvre qui serait inouie et improbable pour l'auteur lui-même. À cet égard, la parole, parole d'autrui ou d'une institution, peu importe, est ce qui vient briser le face-à-face de l'auteur avec lui-même et faire surgir des potentialités que l'auteur ignorait totalement, ce qui va lui permettre d'aller là où il n'aurait jamais été seul. Il y a, du fait de la commande, la possibilité d'une aventure sur des terrains inouïs pour l'auteur, des terrains qui vont s'inventer au fur et à mesure qu'il les parcourt. Car l'écriture est comme une marche, elle est ce qui dessine le paysage qu'elle parcourt. À partir de cette nécessité de la commande, il faudrait établir des régimes de la commande. On peut penser, par exemple, au régime de la commande dans le cadre du mécénat de la Renaissance, époque à laquelle l'artiste se voyait déterminé par toutes les données de la production: il savait à qui était destinée l'œuvre, qui la commandait, quel thème il devait illustrer, avec quels matériaux, pour quelle destination, dans quel lieu et pour quelle somme... Il s'agit de ce que Jacques Rancière appelle le "régime représentatif des $\operatorname{arts}^{3}$ ", lequel cesserait avec le "régime esthétique de l'art » qui configure notre situation. Dans la situation «esthétique », il n'y a plus vraiment d'instances capables de s'adresser à l'artiste. D'abord, parce que la scène culturelle est complètement saturée. La situation est alors celle de l'enfant qui, au moins depuis la naissance, est dans un monde où le langage l'a toujours précédé, car on a toujours été précédé par les mots, au moins entendus comme signifiants (ce sont nos parents qui nous prénomment et la société qui nous nomme). Même avant notre naissance, on a été parlé, peut-être désiré par nos parents, qui ont inventé à notre égard des scénarios où se projetaient leurs désirs. On est donc précédé par le monde des signifiants auxquels il faudra donner sens : nous sommes essentiellement des êtres du retard (Freud-Derrida). C'est la grande tâche de l'expérience que d'accoler des signifiés aux signifiants, l'invention des signifiés devenant la grande nécessité de la formation de soi-même. Par ailleurs, on est aussi précédé par un monde d'œuvres et d'artefacts. Le rapport de Walter Benjamin à son père collectionneur et antiquaire en constitue un bon exemple. Comme lui, on naît toujours dans un monde de collections, au cœur d'une collection. On surgit toujours dans des mondes où d'autres, parents ou sociétés, ont 
toujours déjà constitué des collections ; un peu comme si on passait sa jeunesse dans un magasin de statues et de bibelots, de disques et de reproductions de tableaux. Certes, aujourd'hui, ces collections sont davantage immatérielles: on se retrouve face à des mondes d'archives, des mondes de formes. Alors la tâche imaginative de l'enfant ne va pas consister à produire quelque chose à partir de rien ; elle ne connaît pas l'angoisse de la feuille blanche, mais se trouve plutôt placée devant un monde saturé, devant une muraille de culture. Sa tâche va consister à ouvrir des espaces, des interstices. Benjamin écrivait dans Expérience et pauvreté: " on a bouffé trop de culture ». Face à ce constat, il laissait entrevoir deux solutions : soit celle de la table rase, et les exemples de Benjamin vont du côté de ceux qu'il appelle les «modernes»: Descartes, Loos le Viennois ou Le Corbusier, c'est-à-dire ceux qui partent de rien et sont dans la plus grande pauvreté ; soit, autre solution tout aussi benjaminienne, l'idée que l'imagination a toujours affaire à des formes déjà là, déjà constituées : celles offertes par la culture, et que dès lors la tâche de l'imagination ne va pas consister à mettre en formes des éléments sensibles, mais en partant des formes déjà présentes, fixes et stables, à les dissoudre. L'imagination est réellement créatrice quand elle s'attaque à des formes déjà constituées en les entraînant dans un processus de dissolution, de déformation, qui n'est pas destructeur, mais libérateur. Il ne s'agit pas de ruinifier; il n'y a pas chez Benjamin de mythologie de la ruine. Tout peut devenir matériau pour cette imagination, il en ira de même pour la production artistique: les productions de l'industrie culturelle sont son terreau le plus immédiat. Car il s'agit d'une dissolution de la forme déjà là qui a comme but de faire surgir ce qui restait enkysté en son sein : l' apparition ou le contenu. Ce moment de l'apparition est, écrit Benjamin dans les Essais d'esthétique, "messianique ", ce qui montre bien que le prétendu messianisme de Benjamin n'est pas théologique. C'est l'enfant qui est messianique quand, par rapport à des formes bien constituées, il fait surgir des apparitions. Chez l'adulte, cette imagination n'est plus réellement active, sauf s'il peut répondre à des commandes. La commande devient alors, chez lui, ce qui vient suppléer l'imagination, et devrait permettre à des adultes se trouvant eux aussi face à la muraille culturelle, d'introduire des intervalles, des écarts par où leur plume, leur production, va pouvoir passer. C'est la commande qui va créer de l'espacement, or à notre époque plus personne ne passe de commandes ${ }^{4}$. L'artiste, l'écrivain, le philosophe s'obligent d'une commande qui n'existe pas.

4 Jochen Gerz fait le même constat. Artiste allemand installé en France pendant plus de quarante ans, pays qu'il a quitté pour l'Irlande parce qu'il s'était révélé trop inhospitalier, Gerz développe une œuvre paradoxale sur le terrain de l'histoire de l'Europe et de la politique européenne. En apparence, il prend en charge les victimes de notre histoire contemporaine. En fait, il va au cœur de la question de la commande publique. On pourrait ainsi penser qu'il participe à l'actuel courant de victimisation, mais son geste est tout autre; il dit, à propos des victimes, des disparus : «Ne nous occupons pas d'elles, elles ne sont plus. Occupons-nous de nous. " Déjà l'obélisque évanescente d'Harbourg demandait à ses spectateurs : «Où étiez-vous au moment des faits ${ }^{5}$ ?». Puis, à Sarrebruck, les pavés retournés de la cour du château du Land chaque pavé portant sur sa face enfouie le nom d'un cimetière juif vandalisé par les nazis - continuaient d'être écrasés par les fonctionnaires allemands là où passaient auparavant des membres de la Gestapo ${ }^{6}$. Aujourd'hui, il dit de ce travail qu'il porte sur les limites de l'humour, qu'il pose la question des vivants : "Où sont les vivants?", qu'il faut entendre par : «Y a-t-il encore des vivants?». 
5 À Biron, dans le Périgord, il demandait aux habitants : " Y a-t-il une chose pour laquelle vous accepteriez de sacrifier votre vie? »; «Est-ce que la paix peut générer autant de mémoire que la guerre?».

Et les réponses furent affichées sur le monument aux morts 7 . À de vieilles femmes de Cahors, qui avaient connu la guerre, il demanda de témoigner au moment où se déroulait le procès Papon à Bordeaux ${ }^{8}$. Pourquoi des vieux ? Parce que ces derniers disent ne plus rien avoir à dire : soit qu'ils n'en ont plus le courage, soit qu'ils en ont trop vu, soit qu'ils aient atteint un tel degré de sagesse qu'ils sont au-dessus du discours... À Paris, en 2000, devant Notre-Dame, il incita des clochards - ces disparus de la société, ni morts ni vivants parce qu'invisibles - à prendre la parole et écrire sur le sol afin d'exposer leur pauvreté comme on le ferait des œuvres'. Ce qui provoqua un grand scandale dans le monde institutionnel français de l'art contemporain et la fin de toute commande publique. Ce faisant, il traça le cadre d'un nouveau régime de l'art plus politique, et non pas relationnel.

$6 \mathrm{Au}$ fond, sa production a aussi un autre objectif : réintroduire les vainqueurs sous le joug de la loi d'exposition. Nous sommes les vainqueurs, nous Occidentaux, de toutes les batailles; batailles dont le champ ne fut pas nécessairement ou pas seulement politico-militaire. Car les révolutions du siècle dernier furent essentiellement culturelles, ce qui rejoint l'intuition matricielle de Benjamin. La preuve que nous sommes vainqueurs : nous avons limité, dit-il, l'essentiel de la violence du monde à la télévision. Pour le reste, la pacification est en bonne voie: c'est l'Europe qui se construit pas à pas. Autre preuve : nous aimerions bien passer commande à l'artiste, mais nous n'avons rien de précis à commander; pas de thèmes à représenter. Nos représentants lui disent: «Exprimez-vous, créez quelque chose! Faites de l'art, vous aurez un budget. Mais ce n'est pas à nous de vous dire ce que vous avez à faire. L'art n'est-il pas autonome?»

L'insatisfaction du public devant les «arts contemporains » ne provient pas seulement d'un goût cultivé mis à mal par des productions qui le désorientent, ni du seul spectacle d'une autonomie artistique débordant des limites imprécises. Non, les vainqueurs de la culture (dans tous les sens du terme) savent qu'ils ne peuvent être qu'en apparaissant, mais qu'ils ne sont plus dans la position de commander cette apparition. La question de la commande ne se réduit pas au problème du mécanisme de la décision: "Qui a légitimité pour commander? à qui ? comment choisir? comment "communiquer" ce bon choix? " On assiste à un retournement paradoxal, qui questionne l'essence du pouvoir actuel: c'est dorénavant l'artiste qui doit provoquer la commande. Cela suppose déjà que l'artiste sache qu'on ne produit qu'en fonction d'une commande (parce que cette commande, comme le nom, provient de l'autre) ; que cette commande ne peut être de son fait à lui, artiste ; qu'il ne peut se passer commande à lui-même et que, pourtant, cet autre nécessaire à la commande n'existe pas de fait; qu'il faut se méfier de l'inspiration, de la " création ", des muses... Que lui, l'artiste, doit donc aider l'autre à accoucher d'une commande. Jochen Gerz comme Buren ne se reconnaissent d'ailleurs pas en tant qu'artistes du fait d'une maîtrise, d'un savoir-faire spécifique, même poétique, mais, pour Gerz, dans l'art d'accoucher l'autre d'une commande. Gerz est socratique. Qu'est-ce qui empêche l'autre de passer commande ? C'est qu'il est le vainqueur de la culture et qu'en tant que tel, il peut tout au plus commander à un architecte un musée pour abriter sa collection. L'État - le plus grand des collectionneurs -, les mécènes ne peuvent aller plus loin. C'est qu'il y a un risque qu'ils 
ne peuvent affronter, plus grand que la tentative de l'artiste de répondre à la commande, qui est de s'exposer dans une commande. De s'exposer tout court. De libérer des apparitions.

7 Gerz interroge cette énigme: les collectifs, les représentants politiques, comme à Brême ou à Biron, se dessaisissent de la commande en la relayant par des comités d'experts, de spécialistes, des jurys. Ne peut-on sonder cette incapacité à faire surgir en formant l'hypothèse que ces États, ces collectivités, le public lui-même, tous savent bien que leur prospérité actuelle, leur sécurité, leur bien-être d'Européens reposent sur toute une spectralité qui est là sans être là bien sûr?

Gerz en conclut qu'un monument dédié aux disparus - ceux dont on ne sait ce qu'ils devinrent - serait la pire des choses : une érection, pour quelle vérité ${ }^{10}$ ? Ériger un lieu de mémoire, c'est chastement poser un linge sur ce qu'on veut oublier. Que dire alors du Mémorial de l'Holocauste érigé dans le centre de Berlin par Peter Eisenman?

Pour Gerz, s'il y avait encore commande publique directe, alors elle devrait rester insatisfaite. L'artiste déçoit parce que c'est la commande qui, en elle-même, est la question. Celui qui l'intéresse le plus, de nos jours, ce n'est pas tant celui qui croit pouvoir passer commande, l'honnête homme cultivé, car avec lui il faudrait entrer dans une sorte de maïeutique telle que la pratiquait Platon dans le Ménon: "vous croyez que c'est simple de vouloir passer commande, je vais vous montrer votre impuissance à le faire ». Non : l'ultime commanditaire, c'est précisément celui qui est persuadé qu'il n'aura plus jamais à répondre à aucune commande, celui que rien n'attend, celui dont la destination est au chômage.

9 François Lyotard avait un nom pour cette situation et cette époque : postmodernité. En effet, dans le vestibule d'entrée de l'exposition Les Immatériaux de 1985, un fragment de paroi du temple de Karnak-Nord accueillait le visiteur : la déesse offrant le signe de vie au roi Nectanébo II, dernier pharaon indépendant d'Égypte. Lyotard commentait, dans le catalogue de l'exposition, cette fresque : "Les humains recevaient la vie et le sens: l'âme. Ils devaient la rendre, intacte, perfectionnée. Y a-t-il aujourd'hui quelque chose qui leur soit destiné ? C'est un enjeu majeur de cette manifestation Les Immatériaux ${ }^{11}$. »

Ce public de " chômeurs ", c'est aujourd'hui celui des édifices patrimoniaux, tel l'HôtelDieu de Tonnerre. Classé monument historique, c'est la patrimonialisation qui sauva ce dernier de la destruction, tant la tentation était grande au XIX ${ }^{e}$ siècle de se débarrasser de ces édifices « inutiles ».

\section{BIBLIOGRAPHIE}

Déotte Jean-Louis, Cosmétiques. Simondon, Panofsky, Lyotard, EMSHA, La Plaine Saint-Denis, 2018.

Payot Daniel, La Statue de Heidegger. Art, vérité, souveraineté, Belfort, Éditions Circé, 1998.

À voir

Gerz Jochen, Monument contre le fascisme, 1986-1993. 
— Monument contre le racisme, 1993.

— Le Monument vivant de Biron, 1996.

—Les Témoins, 1998.

— Les Mots de Paris, 2000.

\section{NOTES}

1. Le terme de modernité est si large qu'il ne peut être attribué qu'à des secteurs précis du réel et donc du savoir, ici la peinture. Pour nous, il n'a de pertinence époquale qu'avec l'irruption de l'écriture projective inaugurée par l'appareil perspectif $a u x^{\mathrm{e}}$ siècle à Florence.

2. Ce fut le cas pour Burke et Kant concernant la Révolution française.

3. La notion de représentation chez Jacques Rancière est sociologique et n'entraîne aucune réflexion esthético-philosophique.

4. Sauf l'État, qui passe des commandes pour une statuaire héroïsante toujours datée. C'est l'objet des interventions de l'artiste canadien Peter Gnass.

5. Gerz Jochen, Monument contre le fascisme, 1986-1993.

6. Gerz Jochen, Monument contre le racisme, 1993.

7. Gerz Jochen, Le Monument vivant de Biron, 1996.

8. Gerz Jochen, Les Témoins, 1998.

9. Gerz Jochen, Les Mots de Paris, 2000.

10. Dans La Statue de Heidegger. Art, vérité, souveraineté (Éditions Circé, 1998), Daniel Payot a bien montré, à la suite de Philippe Lacoue-Labarthe, l'importance de la vérité comme stèle et donc comme statue souveraine et irruptive chez Heidegger. Je ne peux que renvoyer à ce commentaire.

11. Voir notre commentaire de cette exposition époquale dans Cosmétiques. Simondon, Panofsky, Lyotard, EMSHA, La Plaine Saint-Denis, 2018. Web. http://books.openedition.org/ emsha/215.

\section{INDEX}

Mots-clés : œuvre d'art, reconnaissance, légitimité, public, commande, exposition

\section{AUTEUR}

\section{JEAN-LOUIS DÉOTTE}

Jean-Louis Déotte a été professeur de philosophie à l'Université Paris VIII, Saint-Denis, coordinateur du thème « Esthétique, arts et industries » à la MSH Paris Nord, directeur de la collection « Esthétiques » chez L'Harmattan et enfin, directeur de la revue Appareil. 